\title{
Interleukin 6, osteoprotegerin, sRANKL and bone metabolism in inflammatory bowel diseases
}

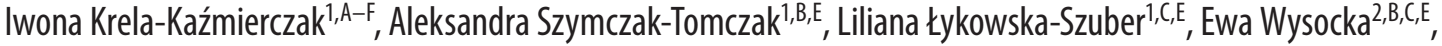 \\ Michał Michalak ${ }^{3, C, E, F}$, Kamila Stawczyk-Eder ${ }^{1, B, D,}$, Katarzyna Waszak ${ }^{1, B, C}$, Krzysztof Linke ${ }^{1, E, F}$, Piotr Eder ${ }^{1, B, C, E, F}$ \\ ${ }^{1}$ Department of Gastroenterology, Dietetics and Internal Medicine, Poznan University of Medical Sciences, Poland \\ 2 Department of Laboratory Diagnostics, Poznan University of Medical Sciences, Poland \\ ${ }^{3}$ Department of Computer Science and Statistics, Poznan University of Medical Sciences, Poland \\ A - research concept and design; $B$ - collection and/or assembly of data; $C$ - data analysis and interpretation; \\ $D$ - writing the article; $E$ - critical revision of the article; $F$ - final approval of the article
}

\author{
Address for correspondence \\ Iwona Krela-Kaźmierczak \\ E-mail:krela@op.pl \\ Funding sources \\ This study was financed from the project \\ of Polish Ministry of Science and Higher \\ Education nr 402481737. \\ Conflict of interest \\ None declared

\section{Acknowledgements} \\ In memory of the late Professor Krzysztof Linke, \\ who supported us with his knowledge, \\ ideas and kindness.
}

Received on December 31, 2016

Reviewed on April 29, 2017

Accepted on July 4, 2017

\begin{abstract}
Background. Cytokines are mediators of inflammatory processes in the course of inflammatory bowel disease (IBD) and participate in the bone metabolism. Interleukin 6 (IL-6) initiates osteoclastogenesis by modulating the activity of soluble receptor activator of nuclear factor kappa B ligand (sRANKL) and osteoprotegerin.
\end{abstract}

Objectives. The aim of the study was to evaluate bone mineral density (BMD) by densitometry and the concentration of interleukin 6, osteoprotegerin (OPG) and SRANKL protein (SRANKL) by ELLSA in patients with IBD in relation to the control group; to assess the relationship between IL-6, OPG, SRANKL and BMD; and to assess the impact of disease duration and number hospitalization on BMD.

Material and methods. The studied group included 37 patients with Crohn's disease (I - CD), 37 patients with ulcerative colitis (II - UC) and 37 healthy subjects - control group (III - CG).

Results. The prevalence of osteoporosis and osteopenia was as follows: in I - CD, 18.92\% and 32.43\% in $L 2-L 4 ; 13.51 \%$ and $35.13 \%$ in the neck, and in $I-U C, 2.7 \%$ and $37.84 \%$ in $L 2-L 4 ; 2.7 \%$, and $29.73 \%$ in the femoral neck. The concentration of IL-6 correlated negatively with T-scores in the neck for the whole group, and in group $\mathrm{I}-\mathrm{CD}$, there was a significant positive correlation between serum OPG and IL-6.

Conclusions. The incidence of osteopenia and osteoporosis in patients with IBD is high and increases with the duration of the disease and the number of hospitalizations. Patients with $C D$ are at a higher risk of skeletal pathology than patients with UC. IL-6 can modulate bone mineral density in the femoral neck especially in the course of $C D$.

Key words: cytokines, osteoporosis, inflammatory bowel disease

DOI

$10.17219 /$ acem $/ 75675$

Copyright

Copyright by Author(s)

This is an article distributed under the terms of the

Creative Commons Attribution Non-Commercial License

(http://creativecommons.org/licenses/by-nc-nd/4.0/) 


\section{Introduction}

Inflammatory bowel diseases (Crohn's disease and ulcerative colitis) cause the development of bone pathologies, like osteopenia and osteoporosis, whose pathogenesis is not fully understood. There are several risk factors for osteoporosis in patients with inflammatory bowel diseases (IBD), including glucocorticoid therapy, reduced physical activity, bone resorption intensified by inflammation (elevated levels of proinflammatory cytokines such as interkeukin-6, interleukin 1 and tumor necrosis factor alpha), poor dietary calcium intake (related to lactose intolerance), magnesium deficiency in the diet, vitamin D deficiency, decreased albumin concentration, and impaired intestinal absorption. ${ }^{1,2}$ Patients with inflammatory bowel diseases, in whom inflammation is quenched, have higher bone mineral density (BMD) in remission. The overall risk of bone fractures in IBD patients, measured as 1 per 100 patientyears, is $40 \%$ higher than in the general population and this risk increases with age. ${ }^{1,3}$ Cytokines are a system that governs the functioning of many systems. They are mediators of inflammatory processes in the course of inflammatory bowel diseases and are involved in resorption and bone formation. The interrelation and regulation of the cytokine pathway may play a role in bone pathology in patients with IBD. Interleukin 6 (IL-6) is one of the pro-inflammatory cytokines that initiates and enhances inflammation, and also stimulates bone resorption by developing osteoclast progenitors. IL- 6 is a glycosylated polypeptide of molecular weight 21-28 kDa, composed of 4 long $\alpha$ helices connected by loops. It is a typical secretory protein, which is produced together with the $\mathrm{N}$-terminal signal peptide. The gene encoding IL- 6 is located on chromosome 7 and contains 5 coding segments (exons). IL- 6 is the main stimulus for the production of most acute phase proteins. ${ }^{4}$ Schulte et al. has demonstrated that the polymorphism of IL-6 does not affect bone loss in patients with IBD. ${ }^{5}$ IL- 6 induces a variety of factors, mainly interleukin-1 (IL-1), but also tumor necrosis factor alpha (TNF- $\alpha) .{ }^{6}$ The gene promoter has sequences that bind transcription factors such as nuclear factor kappa B (NF-k $\beta)$, which regulate gene transcription of IL- 6 in a manner dependent on the cell type and the activating agent. It is worth noting that attempts have already been made to use antibodies against IL-6 in Crohn's disease. ${ }^{7}$ IL- 6 is one of the proinflammatory cytokines that intensifies osteoclastogenesis, modulating activity of soluble receptor activator of nuclear factor kappa $\beta$ ligand (sRANKL), which, contrary to osteoprotegerin, does not have osteogenic effects. ${ }^{8}$ IL- 6 is produced by macrophages, monocytes, endothelial cells, and $\mathrm{T}$ and B lymphocytes. It shows biological activity only when combined with a specific receptor located on the membrane surface of the target cell and it is a potent stimulator of inflammatory processes. The receptor for IL-6 is composed of 2 subunits: glycoproteins of $80 \mathrm{kDa}$ and $130 \mathrm{kDa}$. The importance of the osteoprotegerin-sRANKL system increases, as it can be modulated by cytokines, particularly interleukin-6, the concentration of which in serum correlates negatively with BMD.?

\section{Material and methods}

The study group consisted of 37 patients with CD (I - CD) aged $31.7 \pm 8.0$ years on average, including 15 women and 22 men; 37 patients with ulcerative colitis (II - UC) aged $40.6 \pm 15.1$ years on average, including 21 women and 16 men; and 37 healthy volunteers aged $29.6 \pm 8.0$ years on average, including 18 women and 19 men, who constituted the control group (III - CG). The inclusion criteria were as follows: age between 18 and 60 years, diagnosis of IBD based on cross-sectional imaging and/or endoscopy with histopathological confirmation, disease duration $>1$ year, lack of any other conditions (e.g., rheumatoid arthritis, chronic renal failure), lack of actually biological and steroids therapy which could affect the cytokines profile.

Densitometry of the lumbar spine with L2-L4 assessment and densitometry of the proximal epiphysis of the femur with the assessment of the femoral neck was carried out on all patients, using the Dual Energy X-ray Absorptiometry (DEXA-Lunar DPX-IQ; GE Healthcare Lunar, Boston, USA) technique. The analysis took into account the values of BMD as well as the T-score and Z-score indices. Each patient filled a specially designed questionnaire concerning the current progress and duration of the disease, number of exacerbations and hospitalizations, time and type of pharmacological treatment.

Serum samples for cytokin determinations were stored at $-25^{\circ} \mathrm{C}$ for an average period of 2 months. The samples were not thawed until assay. Quantitative sandwich enzyme immunoassay method (enzyme-linked immunosorbent assay, ELISA) with monoclonal antibody specific for each cytokine/interleukin (IL) specified below was employed. The serum concentration of interleukin 6 was measured by ELISA kits (R\&D Systems Inc., Minneapolis, USA) on microplater reader Sunrise $^{\mathrm{TM}}$ (Tecan Group Ltd., Männedorf, Switzerland), with a sensitivity of $0.70 \mathrm{pg} / \mathrm{mL}$. The following intra-assay and inter-assay coefficient of variations (CV) were calculated for IL-6: $1.8 \%$ and $3.4 \%$. The serum concentrations of sRANKL and OPG were measured by ELISA kits by a sandwich immunoassay method including monoclonal antibodies (BioVendor - Laboratorni medicina a.s., Brno, Czech Republic) on microplater reader Sunrise ${ }^{\mathrm{TM}}$ (Tecan Group Ltd., Männedorf, Switzerland), with a sensitivity of $0.1 \mathrm{pmol} / \mathrm{L}$, while intra-assay and inter-assay coefficient of variations were: $6.5 \%$ and $6.9 \%$ for sRANKL and $6.0 \%$ and $7.2 \%$ for OPG, respectively. Statistical analysis was carried out using the Kruskal-Wallis test with Dunn's post hoc test to distinguish homogeneous groups. The relationship between the analyzed parameters was assessed using Spearman's rank method. The osteopenia and osteoporosis prevalence in analyzed groups was compared 
with test for proportions. The analysis was carried out using STATISTICA PL v. 10 software (StatSoft, Tulsa, USA). All test were considered significant at $\mathrm{p}<0.05$.

Approval for the conduct of the study was obtained from the Bioethics Committee at the Poznan University of Medical Sciences (consent No. 92/09). Informed consent was obtained from every participant.

\section{Results}

The aim of the study was to evaluate bone mineral density (BMD) and the prevalence of osteopenia and osteoporosis and to determine the concentration of interleukin-6, osteoprotegerin (OPG) and sRANKL protein (sRANKL) in patients with inflammatory bowel diseases in relation to the control group, and to assess the relationship between IL- 6 and OPG, RANKL and s-BMD. The research objective was also to assess the impact of disease duration and number of hospitalizations on BMD. Characteristics of Crohn's disease patients (A) ulcerative colitis (UC) and the control group (B) is included in the table (Table 1).

The analyzed groups were not homogeneous with respect of age. The oldest was group of patients with UC (41 years). This group significantly differs from $C D$ (32 years; $\mathrm{p}<0.0001$ ) and control group (30 years; $p=0.0002)$. No significant difference was observed between CD and control group with respect of age.

The characteristics of patients and control groups are presented in Table 1.

The prevalence of osteoporosis and osteopenia in group I - CD and II - UC in $\mathrm{L} 2-\mathrm{L} 4$ and the femoral neck are presented in Table 2.

I - CD patients group is characterized by a significantly higher rate of osteoporosis compared to II - UC patients group $18.92 \%$ vs $2.7 \%, \mathrm{p}=0.0247$. Neck BMD and T-score in I - CD group differs significantly from III - CG group $(\mathrm{p}<0.05=0.0007)$, but is not significantly different from II - UC group. The mean concentrations of IL-6 (pg/mL), OPG (pmol/L) and sRANKL (pmol/L) of patients and control groups are presented in Table 3.

The level of OPG (pmol/L) differed significantly between all analyzed groups. Thehighest level was observed in the I-CD patients group. For both I - CD and II - UC group the IL-6 (pg/mL) level was significantly higher compared to III - CG (I - CD vs III - CG group p < 0.0001; II - UC vs III - CG p < 0.0001). No significant differences in IL-6 (pg/mL) level were observed between the I - CD and the II - UC group (Table 3).

The concentration of IL- 6 correlated negatively with neck T-scores for the whole group $(\mathrm{r}=-0.33 ; \mathrm{p}=0.0004)$, and there was a significant positive correlation $(r=0.51$; $\mathrm{p}=0.0017$ ) between OPG and IL-6 in group I - CD. In patients with CD and UC, the mean concentrations of IL-6 were higher than in CG. This difference was statistically significant $(\mathrm{p}<0.0001)$. There was no significant difference in disease duration between analyzed groups $8.05 \pm 5.29$ in group I - CD, and $8.03 \pm 7.92$ in group II - UC. Disease duration correlated with neck $\mathrm{T}$ - and $\mathrm{Z}$-scores $(\mathrm{r}=-0.40$; $\mathrm{p}<0.0001$ and $\mathrm{r}=-0.24 ; \mathrm{p}=0.0120)$. A similar correlation
Table 1. Characteristics of Crohn's disease patients, ulcerative colitis patients and control group parameters values presented as means and standard deviations

\begin{tabular}{|l|c|c|c|c|}
\hline \multirow{2}{*}{\multicolumn{1}{|c|}{ Parameters }} & $\begin{array}{c}\text { Crohn's disease } \\
n=37\end{array}$ & $\begin{array}{c}\text { Ulcerative colitis } \\
n=37\end{array}$ & $\begin{array}{c}\text { Control group } \\
n=37\end{array}$ & \multirow{2}{*}{ p-value } \\
\cline { 2 - 5 } & mean \pm SD & mean \pm SD & mean $\pm S D$ & \\
\hline Age [years] & $31.76 \pm 8.06^{\mathrm{a}, \mathrm{b}}$ & $40.65 \pm 15.11^{\mathrm{b}}$ & $29.57 \pm 8.01^{\mathrm{a}}$ & 0.0014 \\
\hline Weight [kg] & $62.81 \pm 13.8^{\mathrm{a}}$ & $66.89 \pm 15.3^{\mathrm{a}, \mathrm{b}}$ & $74.57 \pm 14.61^{\mathrm{b}}$ & 0.0054 \\
\hline BMI & $20.98 \pm 3.26^{\mathrm{a}}$ & $23.05 \pm 4.26^{\mathrm{a}, \mathrm{b}}$ & $24.74 \pm 3.54^{\mathrm{b}}$ & 0.0001 \\
\hline Disease duration [years] & $8.03 \pm 7.92$ & $8.05 \pm 5.29$ & - & $0.4174^{*}$ \\
\hline L2-L4 BMD & $1.11 \pm 0.19^{\mathrm{a}}$ & $1.17 \pm 0.16^{\mathrm{a}, \mathrm{b}}$ & $1.22 \pm 0.08^{\mathrm{b}}$ & 0.0074 \\
\hline T-score L2-L4 & $-0.95 \pm 1.56^{\mathrm{a}}$ & $-0.42 \pm 1.2^{\mathrm{a}, \mathrm{b}}$ & $0.11 \pm 0.72^{\mathrm{b}}$ & 0.0030 \\
\hline Z-score L2-L4 & $-0.42 \pm 1.41$ & $-0.03 \pm 1.28$ & $0.04 \pm 0.66$ & 0.1152 \\
\hline Neck BMD & $0.92 \pm 0.2^{\mathrm{a}}$ & $0.97 \pm 0.16^{\mathrm{a}}$ & $1.09 \pm 0.16^{\mathrm{b}}$ & 0.0007 \\
\hline T-score neck & $-0.86 \pm 1.49^{\mathrm{a}}$ & $-0.44 \pm 1.19^{\mathrm{a}}$ & $0.49 \pm 1.04^{\mathrm{b}}$ & 0.0001 \\
\hline Z-score neck & $-0.41 \pm 1.28^{\mathrm{a}}$ & $0.03 \pm 1.1^{\mathrm{a}, \mathrm{b}}$ & $0.42 \pm 0.99^{\mathrm{b}}$ & 0.0068 \\
\hline
\end{tabular}

a, b - groups followed by the same letter do not differ statistically significantly; SD - standard deviation; BMI - body mass index; * Mann-Whitney U test.

Table 2. Prevalence of osteoporosis and osteopenia in IBD (CD, UC)

\begin{tabular}{|c|c|c|c|c|c|c|}
\hline \multirow{2}{*}{ Prevalence } & $C D$ & UC & \multirow{2}{*}{$p$-value } & $C D$ & UC & \multirow{2}{*}{$p$-value } \\
\hline & \multicolumn{2}{|c|}{ L2-L4 } & & \multicolumn{2}{|c|}{ neck } & \\
\hline Osteoporosis [\%] & 18.92 & 2.7 & 0.0247 & 13.51 & 2.7 & 0.0884 \\
\hline Osteopenia [\%] & 32.43 & 37.84 & 0.6260 & 35.13 & 29.73 & 0.6198 \\
\hline
\end{tabular}

IBD - bone mineral density; CD - Crohn's disease; UC - ulcerative colitis.

Table 3. Mean serum concentrations of tested cytokines, Crohn's disease, ulcerative colitis and control group (comparison of the studied groups)

\begin{tabular}{|l|c|c|c|c|}
\multirow{2}{*}{\multicolumn{1}{|c|}{ Cytokine }} & $\begin{array}{c}\text { Crohn's disease } \\
\text { group } n=37\end{array}$ & $\begin{array}{c}\text { Ulcerative colitis } \\
n=37\end{array}$ & $\begin{array}{c}\text { Control group } \\
n=37\end{array}$ & \multirow{2}{*}{ p-value } \\
\cline { 2 - 5 } & $($ mean \pm SD) & $($ mean \pm SD) & (mean \pm SD) & \\
\hline IL-6 [pg/mL] & $6.73 \pm 5.23^{\mathrm{b}}$ & $4.93 \pm 4.61^{\mathrm{b}}$ & $1.41 \pm 1.32^{\mathrm{a}}$ & $<0.0001$ \\
\hline OPG $[\mathrm{pmol} / \mathrm{L}]$ & $8.76 \pm 3.22^{\mathrm{a} . \mathrm{b}}$ & $6.02 \pm 2.51^{\mathrm{a}}$ & $9.42 \pm 2.01^{\mathrm{b}}$ & $<0.0001$ \\
\hline sRANKL $[\mathrm{pmol} / \mathrm{L}]$ & $284.87 \pm 213.05$ & $223.82 \pm 118.14$ & $236.84 \pm 111.63$ & 0.5856 \\
\hline
\end{tabular}

a, b groups followed by the same letter do not differ statistically significantly; SD - standard deviation; OPG - osteoprotegerin; sRANKL - soluble receptor activator of nuclear factor kappa B ligand. 
has been demonstrated for the number of hospitalizations - T-score $(\mathrm{r}=-0.41, \mathrm{p}<0.0001)$ and Z-score $(\mathrm{r}=-0.29$, $\mathrm{p}=0.0027)$, respectively.

\section{Discussion}

The study shows that the incidence of osteoporosis and osteopenia in Polish patients with IBD is high, just as in other study populations; however, further population research is needed. The incidence of osteoporosis is greater in patients with CD than in UC patients, as evidenced by other researchers. ${ }^{9}$ However, no statistically significant differences in BMD between the CD and UC groups have been demonstrated. A similar incidence of osteoporosis and osteopenia has been described in a Tunisian population of patients with IBD. Neck osteoporosis was observed in $31.8 \%$ of patients with CD and $13 \%$ of UC subjects, while L2-L4 osteoporosis was found in 40.9\% of $\mathrm{CD}$ and $8.7 \%$ of $\mathrm{UC}$ patients, respectively. The incidence of neck osteoporosis was higher in CD patients. A study of 200 IBD patients in the Iranian population demonstrated an equally high incidence of osteoporosis (24.1\%) and osteopenia (50.3\%). ${ }^{10}$ The loss of bone mass in the course of IBD is regulated by immune system mediators, such as proinflammatory cytokines (TNF- $\alpha$, IL-1, IL-6, IFN- - ). Other TNF-related cytokines, such as RANK/RANKL/ OPG are important mediators of inflammation in the intestine, and they participate in the pathophysiology of bone loss leading to the development of osteoporosis. There are certainly many mechanisms that lead to the development of osteoporosis in the course of IBD, but inflammation is a very important factor. The key mediator of inflammation in IBD is TNF- $\alpha$, also involved in the inflammatory process in the intestine and bone metabolism, thus it can be hypothesized that TNF- $\alpha$ neutralizing therapy can improve the therapeutic strategies in the treatment of secondary osteoporosis associated with inflammation also in the course of IBD. ${ }^{11-13}$ IL-6, whose levels increase with the severity of inflammation in the course of IBD, as a factor in osteoclastogenesis may affect the OPG/RANKL pathway. Further research is needed to confirm the effect of proinflammatory cytokines on the OPG/RANKL pathway in the course of IBD and thus to investigate the effect of these cytokines on bone loss and osteoporosis in the course of these diseases. ${ }^{12,13}$ Interleukin 6 promotes destructive processes, affecting osteoclastogenesis. Interleukin 6 , as well as IL-1 3 , TNF- $\alpha$ and IL-17, stimulates the production of cytokine RANKL, increases its cellular expression and induces bone resorption. ${ }^{14}$

Our study has also several limitations. The most important one is that we did not perform an association analysis between bone mineral density and the severity of the disease flare. Nevertheless, since all patients enrolled into the study had active disease, we decided not to divide the study group into another clinical subcategories in order to obtain more conclusive results. Our results are different as they indicate that OPG may be stimulated by IL-6. This relationship, therefore, requires broader research, perhaps in larger groups of patients. Experimental studies raise the importance of proinflammatory cytokines including IL-6, IL-1 and TNF- $\alpha$ as important factors that regulate bone resorption and may play a role in bone loss associated with age, estrogen deficiency (postmenopausal osteoporosis), and in the course of IBD. However, further research in this area is necessary. ${ }^{15}$ According to other authors, the concentrations of IL- 6 in patients with IBD and osteoporosis are increased, and it is thus believed that IL- 6 plays a role in the pathogenesis of bone loss, but the mechanism is not fully understood and requires further studies. ${ }^{16}$ The findings suggest that the concentration of IL- 6 in the serum of patients with IBD in the Croatian population is a clinically important parameter and correlates with the activity of inflammatory disease. ${ }^{17}$ IL-6, as a pro-inflammatory cytokine, adversely affects bone turnover by influencing the activity of osteoclasts. The negative effect of IL- 6 on bone tissue has been shown in patients with rheumatoid arthritis. ${ }^{18}$ An increased activity of IL- 6 accelerates the process of bone resorption through the activity of osteoclasts. Other authors indicate that IL- 6 concentrations were higher in postmenopausal women with osteoporosis than in the control group. This allows for the conclusion that this cytokine has destructive effects on bone tissue. ${ }^{19,20}$ Our results are similar to the study conducted by Polinska et al. ${ }^{21}$ The study demonstrated that the serum concentrations of IL- 6 in UC was 3 times higher than in healthy group. Clinical studies of tocilizumab, a humanized monoclonal antibody against IL-6 receptors, have shown its high efficiency in the treatment of rheumatoid arthritis. ${ }^{22,23}$ Therefore, these studies may contribute to the development and implementation of new secondary osteoporosis therapy in the course of IBD.

\section{Conclusions}

The incidence of osteopenia and osteoporosis in patients with inflammatory bowel diseases is high and increases with the duration of the disease and the number of hospitalizations. Patients with Crohn's disease are at a higher risk of skeletal pathology than patients with ulcerative colitis. Interleukin 6, as a proinflammatory cytokine, can modulate bone mineral density in the femoral neck, which can cause a loss of bone mass, especially in the course of Crohn's disease. The effect of interleukin 6, which modulates the OPG/ sRANKL system, on bone mass density in the course of inflammatory bowel diseases requires further study.

\section{References}

1. Levine JS, Burakoff R. Extraintestinal manifestations of inflammatory bowel disease. Gastroenterol Hepatol. 2011;7(4):235-241.

2. Krela-Kaźmierczak I, Szymczak A, Łykowska-Szuber L, et al. Osteoporosis in gastrointestinal diseases. Adv Clin Exp Med. 2016;1: 185-190. 
3. American Gastroenterological Association medical position statement: Guidelines on osteoporosis in gastrointestinal diseases. Gastroenterology. 2003;124:791-794.

4. Ishihara K, Hirano T. IL-6 in autoimmune disease and chronic inflammatory proliferative disease. Cytokine Growth Factor Rev. 2002;13:357-368.

5. Schulte CMS, Goebell H, Rõher HD, et al. Genetic determinants of IL-6 expression levels do not influence bone loss in inflammatory bowel disease. Dig Dis Sci. 2001;46(11):2521-2528.

6. Park JY, Pillinger MH. Interleukin- 6 in the pathogenesis of rheumatoid arthritis. Bull NYU Hosp Jt Dis. 2007;65(1):4-10.

7. Ito $\mathrm{H}$. Novel therapy for Crohn's disease targeting IL-6 signaling. Expert Opin Ther Targets. 2000;8(4):287-294.

8. Sylvester AF. Effects of inflammatory bowel diseases on bone metabolism. IBMS Bonekey. 2009;6(11):420-428.

9. Boubaker J, Feki M, Hsairi M, et al. Osteoporosis and inflammatory bowel disease: Prevalence and risk factors in Tunisian patients. Gastroenterol Clin Biol. 2003;27(10):901-907.

10. Shirazi KM, Somi MH, Rezaeifar P, et al. Bone density and bone metabolism in patients with inflammatory bowel disease. Saudi J Gastroenterol. 2012;18(4):241-247.

11. Tilg H, Moschen AR, Kaser A, et al. Gut, inflammation and osteoporosis: Basic and clinical concepts. Gut. 2008;57(5):684-694.

12. Miheller P, Muzes G, Racz K, et al. Changes of OPG and RANKL concentrations in Crohn's disease after infliximab therapy. Inflamm Bowel Dis. 2007;13(11):1379-1384.

13. Ali T, Lam D, Bronze MS, et al. Osteoporosis in inflammatory bowel disease. Am J Med. 2009;122(7):599-604.

14. Hashizume M, Hayakawa N, Mihara M. IL-6 trans-signaling directly induces RANKL on fibroblast-like synovial cells and is involved in RANKL induction by TNF- $a$ and IL-17. Rheumatology. 2008;47(11):1635-1640.

15. McLean RR. Proinflammatory cytokines and osteoporosis. Curr Osteoporos Rep. 2009;7(4):134-139.

16. Pollak RD, Karmeli F, Eliakim R, et al. Femoral neck osteopenia in patients with inflammatory bowel disease. Am J Gastroenterol. 1998;93(9):1483-1490.

17. Takac B, Mihaljevic S, Stefanić M, et al. Importance of interleukin 6 in pathogenesis of inflammatory bowel disease. Coll Antropol. 2014;38(2):659-664.

18. Manolagas SC. The role of cytokines and their receptors in bone. Ann NY Acad Sci. 1998;840:194-204.

19. Romas E, Martin TJ. Cytokines in the pathogenesis of osteoporosis Osteoporos Int. 1997;7:47-53.

20. Keen RW, Woodford-Richens KL, Lanchbury JS, et al. Allelic variation at the interleukin-1 receptor antagonist gene is associated with early postmenopausal bone loss at the spine. Bone. 1998;23:367-371.

21. Polinska B, Matowicka-Karna J, Kemona H. Assessment of the influence of the inflammatory process on the activation of blood platelets and morphological parameters in patients with ulcerative colitis (colitis ulcerosa). Folia Histochem Cytobiol. 2011;49:119-124

22. Allocca M, Jovani M, Fiorino G, et al. Anti-IL-6 treatment for inflammatory bowel diseases: Next cytokine, next target. Curr Drug Targets. 2013;14(12):1508-1521.

23. Kang S, Tanaka T, Kishimoto T. Therapeutic uses of anti-interleukin-6 receptor antibody. Int Immunol. 2015;27(1):21-29. doi:10.1093/ intimm/dxu081 